\title{
Capacity Enhancing Techniques for High Throughput Satellite Communications
}

Svilen Dimitrov ${ }^{1}$, Stefan Erl $^{1}$, Benjamin Barth ${ }^{1}$, Rosalba Suffritti ${ }^{2}$, Niccoló

Privitera $^{2}$, Gabriele Boccolini ${ }^{3}$, Adegbenga B. Awoseyila ${ }^{4}$, Argyrios

Kyrgiazos $^{4}$, Barry G. Evans ${ }^{4}$, Stephan Jaeckel ${ }^{5}$, Belén Sánchez ${ }^{6}$, Ana Yun

Garcia $^{6}$, and Oriol Vidal ${ }^{7}$ *

1 German Aerospace Center (DLR), Satellite Networks Department, 82234 Wessling,

Germany, \{svilen.dimitrov, stefan.erl, benjamin.barth\}@dlr.de,

2 Mavigex S.r.l., ICT Solutions, 40125 Bologna, Italy,

\{rsuffritti, niccolo.privitera\}@mavigex.com

${ }^{3}$ Galician R\&D Center in Advanced Telecommunications (GRADIANT), 36310

Vigo, Spain, gboccolini@gradiant.org

4 University of Surrey, Centre for Communication Systems Research, GU27XH, UK,

\{a.awoseyila, a.kyrgiazos, b.evans\}@surrey.ac.uk

5 Fraunhofer Heinrich Hertz Institute, 10587 Berlin, Germany, stephan.jaeckel@hhi.fraunhofer.de

6 Thales Alenia Space Espana, Madrid, Spain,

\{belen.sanchez, ana.yungarcia\}@thalesaleniaspace.com

7 Airbus Defence \& Space, Telecom Systems Department, Toulouse, France,

oriol.vidal@astrium.eads.net

\begin{abstract}
In this paper, we present physical layer and system level techniques that can increase the capacity of a multi-beam high throughput satellite (HTS) communication system. These include advanced predistortion and equalization techniques for the forward link, synchronization and non-linear distortion minimization techniques for the return link waveforms. Interference management techniques such as precoding, multi-user detection (MUD), interference cancellation and coordination are evaluated under realistic co-channel interference (CCI) conditions. Interference-aware radio resource management (RRM) algorithms are presented for the forward and return links with full frequency reuse. These include satellite-switched smart gateway diversity, interferenceaware scheduling (IAS) for the forward link and scheduling based on multi-partite graph matching for the return link. The capacity enhancing techniques are evaluated in the Broadband Access via Integrated Terrestrial and Satellite Systems (BATS) framework.
\end{abstract}

Key words: Satellite communications, interference management, radio resource management, spectral and power efficiencies.

\footnotetext{
* This paper has been accepted for publication in the Proceedings of the of International Conference on Wireless and Satellite Systems 2015 (WiSATS 2015), 6-7 July 2015, Bradford, UK, DOI: 10.1007/978-3-319-25479-1_6. (C)2015 ICST.
} 


\section{Introduction}

In order to serve the increasing demand for satellite broadband [1], a multi-beam HTS system needs to employ a very high resource reuse over the coverage area, combined with intelligent interference management and resource management mechanisms to alleviate the resulting CCI. In addition, the utilization of higher pieces of bandwidth, for example in the Ka band, requires the application of suitable signal processing techniques and waveforms, in order to efficiently manage the interference in the physical layer.

In this paper, non-linear pre-distortion and equalization techniques are evaluated in a multi-carrier scenario in the forward satellite link. In addition, fastFourier-transform-(FFT)-based waveforms, such as single-carrier frequency division multiple access (SC-FDMA), orthogonal FDMA (OFDMA) and filterbank multi-carrier (FBMC) are compared in a return link setup in terms of power and spectral efficiencies, and suitable synchronization algorithms for the satellite channel are proposed.

In addition, interference management techniques are evaluated under realistic CCI conditions. Techniques such as precoding, multi-user detection, interference cancellation and interference coordination allow the use of more aggressive frequency reuse patterns as compared to conventional frequency reuse 4. Significant gains in terms of available system bandwidth and total throughput are presented, in particular when multi-gateway cooperation strategies are considered.

The fixed superframe length introduced with the DVB-S2X standard allows for burst mode transmission, as is the case with the return link DVB-RCS2 standard. While the return link inherently benefits from RRM and scheduling to mitigate the CCI, the forward link can only benefit, if a number of the interfering beams are switched off at a given time step or if the system is not under full load. In this paper, the performance of several RRM solutions is evaluated in the forward and return satellite links of a practical HTS system.

The rest of the paper is organized as follows. Section 2 presents the study of physical layer techniques. Section 3 elaborates on interference management techniques, while Section 4 presents the radio resource management algorithms. Finally, Section 5 concludes the paper.

\section{Waveform}

In the forward link DVB-S2X standard, static predistortion is applied at the gateway to the time division multiplexing (TDM) waveform to compensate the constellation warping due to the nonlinearities in the channel. In addition, a linear equalizer is employed at the user terminal to counter both linear and nonlinear distortion. However, the evaluation of more potent compensation techniques, such as memory polynomials predistortion and equalization [2], for the newly introduced roll-off factors of $10 \%$ and $5 \%$, is still an open issue.

In this paper, we also study the application of FFT-based waveforms in the return link and compare their power and spectral efficiencies with time division 


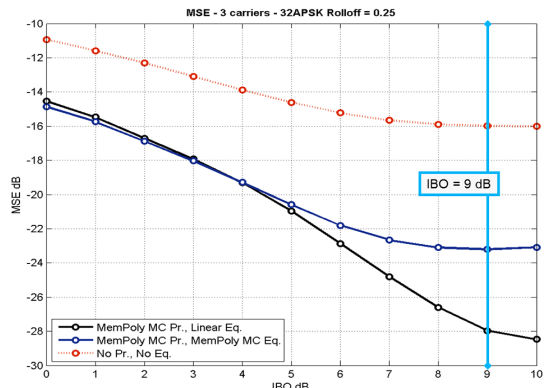

(a)

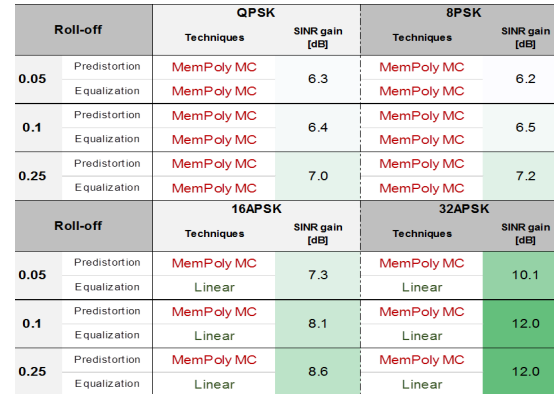

(b)

Fig. 1: a) MSE vs. IBO for advanced predistortion/equalization, 32-APSK, 3 carriers, 0.25 roll-off. b) Non-linear compensation techniques and MSE gains.

multiple-access (TDMA) with $5 \%$ and $20 \%$ roll-offs. The FFT-based waveforms depend strongly on frame timing and carrier frequency synchronization of all users in order to mitigate inter-symbol interference (ISI), inter-carrier interference (ICI) and multiple access interference (MAI).

\subsection{Non-linear compensation by predistortion and equalization in forward link}

In a multi-carrier satellite forward link, predistortion and equalization algorithms are applied jointly to more than one carrier with the goal of exploiting mutual correlation between the carriers that share the same satellite transponder. The mean-squared error (MSE) performance for 32-APSK is presented in Fig. 1 a). The optimum combination of non-linear predistortion and equalization has been selected on the basis on the MSE results w.r.t the baseline scenario without predistortion and equalization. The analysis has been performed for roll-off values in the set of $[0.25,0.10,0.05]$. The MSE improvements at the modulation-dependent working input back-off (IBO) have been computed, and the most promising techniques are summarized in Fig. 1 b).

\subsection{Synchronization acquisition in return link}

Synchronization acquisition focuses mainly on frame timing at log-on to correct for differential propagation delays in the presence of residual frequency offsets and amplifier nonlinearity. Timing alignment of the uplink transmissions is achieved by applying a timing advance at each user terminal [3]. However, the propagation delays in the satellite channel with round-trip time (RTT) of $500 \mathrm{~ms}$ and differential propagation delays within a spot beam in the order of $\mathrm{ms}$, as compared to an order of $\mu \mathrm{s}$ in a terrestrial link, are considerably larger. Given a typical symbol duration of an FFT-based waveform in the order of $\mu \mathrm{s}$, a guard time greater than the satellite RTT or even the maximum differential 


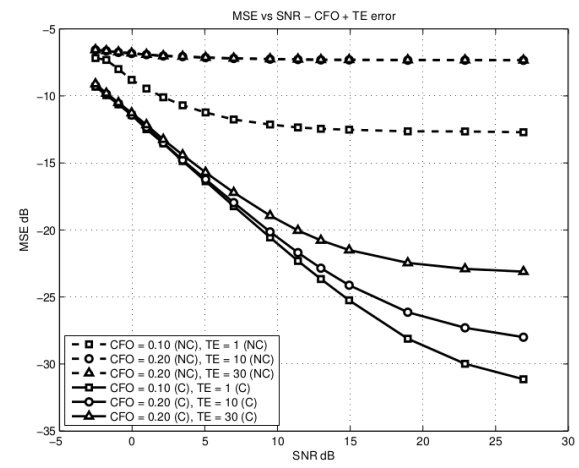

Fig. 2: MSE vs. SNR, CFO and TE compensation. NC stands for "Not Compensated", while C denotes "Compensated".

propagation delay is not affordable. In addition, the cyclic prefix (CP) cannot be dimensioned for using cyclic shifts of a Zadoff-Chu (ZC) sequence.

In order to solve these problems, we propose the use of global-positioningsystem-(GPS)-based timing pre-compensation. A GPS device at each return channel via satellite terminal (RCST) can track its location. This can be combined with existing satellite and network control center (NCC) data to calculate the two-way propagation delay and pre-compensate it before transmitting the random access (RA) preamble. In this way, the timing misalignment between RCSTs can be reduced from ms to ns, resulting in a very low CP overhead.

\subsection{Synchronization tracking and estimation in return link}

After synchronization is acquired, estimation of residual timing error (TE) and carrier frequency offset (CFO) is used for fine synchronization and tracking. The Mengali and Morelli (M\&M) algorithm [4] is chosen for tracking timing offset since it: 1) does not show a definite threshold effect in terms of signalto-noise ratio (SNR); 2) requires low complexity due to the adoption of two implementation strategies, one for small number of points and the other for larger sets; 3) has an estimation range independent from the number of points in the observed sequence. The Moose algorithm [5] is selected for tracking frequency offsets due to its closeness to the Cramer-Rao bound. The remaining static phase offset can be recovered using phase locked loop (PLL) filters before the signal is passed to the demodulator [6], [7].

The algorithms are tested in the SC-FDMA setup with 128 subcarriers in the frame with 16 subcarriers per user. A pilot period of 10 SC-FDMA symbols and a CP of 16 samples are considered. Fig. 2 shows the MSE performance for different values of CFO), TE and SNR. A normalized CFO of 0.1 means that terminal and gateway oscillators are misaligned by $0.1 * \Delta f$, where $\Delta f$ is the subcarrier spacing. TE of 1 means that the acquisition stage outputs a signal with a relative drift of 1 sample w.r.t. the nominal start of frame. The M\&M 


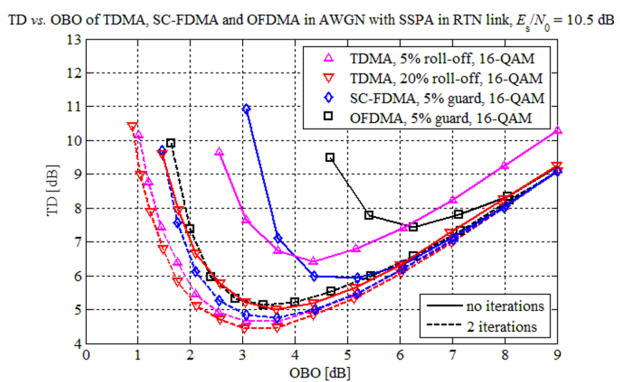

(a) TD of 16-QAM.

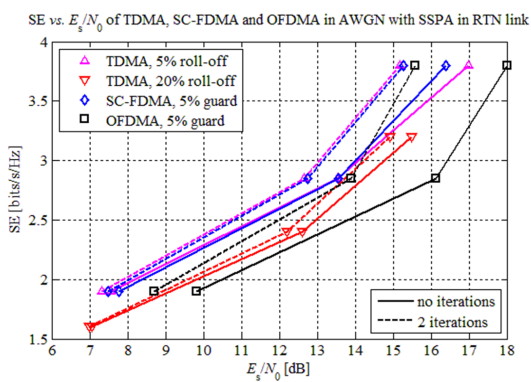

(b) Spectral and power efficiencies.

Fig. 3: Power and spectral efficiencies of TDMA, OFDMA and SC-FDMA in return link.

and Moose algorithms present sufficiently low MSE at low SNR below $0 \mathrm{~dB}$, and the performance is degraded only when the TE approaches the size of the CP.

\subsection{Power efficiency optimization in return link}

The power efficiency of the transmission schemes can be maximized by optimization of output back-off (OBO) and minimization of total degradation (TD) in the non-linear channel. In this study, the SNR requirement and spectral efficiency (SE) are compared for SC-FDMA, OFDMA and state-of-the-art TDMA (with $5 \%$ and $20 \%$ roll-off) with QPSK, 8-PSK and 16-QAM for a practical Ka-band amplifier model. In OFDMA and SC-FDMA, 2048 digital subcarriers per physical carrier are allocated to 32 users with 64 subcarriers per user. In addition, a $5 \%$ guard band is included between the physical carriers [3]. An IMI cancelling receiver is studied, where an estimate of the intermodulation interference (IMI) is calculated and subtracted from the received signal in an iterative fashion [8].

In order to quantify the power efficiency penalty induced by the amplifier nonlinearity, the TD metric for a target bit-error ratio (BER) is defined as follows:

$$
\mathrm{TD}[\mathrm{dB}]=\mathrm{OBO}+\mathrm{SNR}_{\substack{\text { req.non-linear } \\ \text { channel }}}-\mathrm{SNR}_{\substack{\text { req.linear } \\ \text { channel }}} .
$$

Results for the TD of 16-QAM and the SE of the DVB-RCS2 waveforms are presented in Fig. 3. The chosen reference SNR targets in the linear channel are $3.7 \mathrm{~dB}, 8.9 \mathrm{~dB}$ and $10.5 \mathrm{~dB}$, respectively, for a BER target of $10^{-3}$. The gains in power efficiency for the three modulation orders with 2 iterations of IMI cancellation can be summarized as follows: $0.1-0.7 \mathrm{~dB}$ for TDMA with $20 \%$ roll-off, $0.2-1.8 \mathrm{~dB}$ for TDMA with $5 \%$ roll-off, $0.3-1.1 \mathrm{~dB}$ for SC-FDMA and $1.1-2.5 \mathrm{~dB}$ for OFDMA. SC-FDMA demonstrates an overall improvement of the power efficiency of more than $2 \mathrm{~dB}$ as compared to state-of-the-art TDMA with $20 \%$ roll-off. 


\begin{tabular}{|lcccccc|}
\hline & GB & GB & $N_{\mathrm{u}}$ & Eff. & $N_{\mathrm{u}}$ & Eff. \\
& carriers & users & $1 \mathrm{RB}$ & $1 \mathrm{RB}$ & $10 \mathrm{RB}$ & $10 \mathrm{RB}$ \\
\hline OFDMA & $5 \%$ & 0 SC & 162 & $95 \%$ & 16 & $95 \%$ \\
OFDMA & $5 \%$ & 1 SC & 149 & $87 \%$ & 16 & $94 \%$ \\
OFDMA & $5 \%$ & 2 SC & 139 & $81 \%$ & 15 & $93 \%$ \\
SMT & $2 \%$ & 1 SC & 154 & $91 \%$ & 16 & $97 \%$ \\
\hline
\end{tabular}

Table 1: Comparing efficiencies of FBMC (SMT) and OFDM.

\subsection{Spectral efficiency improvement in return link}

In the return link, the received signal is the sum of all user contributions, each one with its own synchronisation offsets and non-linear distortion. These impairments can be mitigated with the insertion of guard band (GB) between users. The SE can be improved by the use of FBMC with staggered multitone (SMT). In OFDMA, $5 \%$ of GB between physical carriers is included. In FBMC, $2 \%$ of GB is considered to take into account non-linear distortions. TABLE 1 shows the efficiency in terms of percentage of subcarriers used for data over the total number of 2048 subcarriers. With 1 resource block (RB) of 12 subcarriers assigned to each user, the best efficiency is achieved by OFDMA without GB. However, as soon as the number of GB subcarriers is increased to reduce the effect of synchronization errors, SMT turns out to be more efficient than OFDMA. SMT efficiency increases, when a larger number of RBs is allocated to each user as reported in the table for $10 \mathrm{RBs}$.

\section{Interference management}

A typical multi-beam satellite system relies on reusing the available frequency bands multiple times across the antenna beams. In such a scenario, the interference introduced by nonorthogonality between beam patterns represents an important issue to be solved in order to improve the capacity of the satellite network. The considered system model is based on a set of realistic data on a multi-beam satellite network covering the European region, composed of 302 spot beams of 0.21 degrees in beamwidth, and operating in Ka-band. Frequency reuse 4 (FR-4) represents the benchmark scenario for the forward and return links of the considered system that foresees two options for the feeder link: the $\mathrm{Q} / \mathrm{V}$ feeder link and the optical feeder link, where in both cases $1.5 \mathrm{GHz}$ of spectrum is allocated in the forward link and $500 \mathrm{MHz}$ in the return link. In addition to FR-4, also FR-2 and FR-1 schemes have been considered. The beams have been grouped in clusters and every cluster is served by one gateway (GW). GWs addressing different clusters can also cooperate to apply jointly the interference management techniques to the entire set of considered beams, such as in an optical feeder link scenario. 


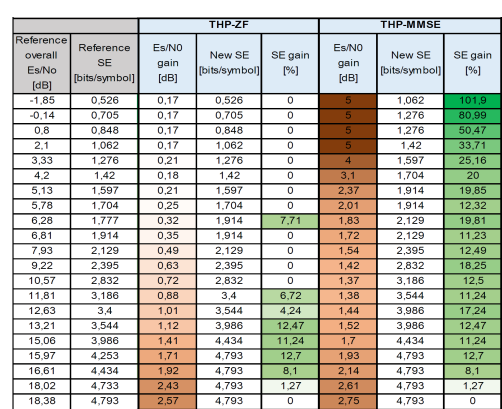

(a)

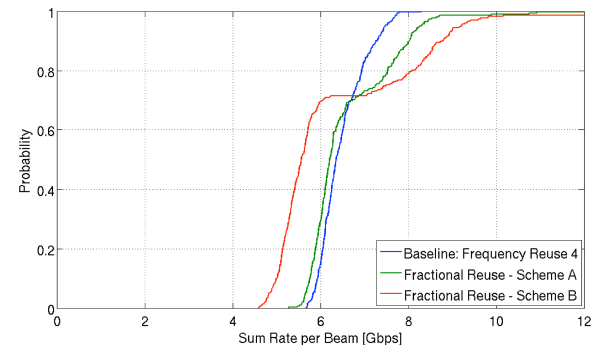

(b)

Fig. 4: a) Es/N0 and SE improvement with THP precoding, FR-4, 4 cooperating GWs. b) Achievable sum rate for different reuse schemes.

\subsection{Forward link}

The interference management schemes in the forward link can be applied at the transmitting GW. The signals referring to the same cluster can be combined using, for example, precoding algorithms before transmission over the uplink to minimize the distortions of every user-received signal resulting from nonorthogonality between the antenna beams. In addition, fractional frequency reuse (FFR) can be also taken into account as a smart resource reuse strategy. In the following, more details on each technique are provided along with the main outcomes of the performance assessment.

Precoding: Linear and non-linear precoding algorithms have been analysed and compared in the considered scenario [9]. The most promising results come from non-linear techniques such as Tomlinson-Harashima Precoding (THP) which uses modulo arithmetic in order to jointly achieve pre-equalization and power limitation of the transmitted signal [10]. The results show that increasing the collaboration between gateways up to the entire set of gateways (optical feeder link scenario) leads to complete cancellation of the interference [9]. The gains in terms of SE and s/N0 are presented in Fig. 4 a) for the case of 4 gateway cooperation.

Fractional frequency reuse: Two FFR schemes, A and B, have been evaluated [11] and compared with FR-4 which reuses $50 \%$ of the total bandwidth in each beam. This is increased to $75 \%$ in FFR scheme A, where $50 \%$ are reused in the beam centres and $25 \%$ are exclusively used for the beam edge. In the FFR scheme B, the full bandwidth is reused: $50 \%$ in the beam centres and $50 \%$ in the beam edges. The CDF of the sum rates is presented in Fig. 4 b). FFR strongly increases the maximum rate. However, the results also indicate a strong impact of the utilized beam patterns on the overall system performance, since the beam patterns have been optimized for FR-4. In order to show the anticipated gain of FFR in the satellite system, the power difference between beam center and beam edge (currently $3 \mathrm{~dB}$ ) needs to be increased. Since the shape of the beam 
pattern is determined by the antenna, a reorganization of the beam layout is advisable in order to maximize the throughput of FFR.

\subsection{Return link}

In a multi-user system, a suboptimal approach is to detect each user independently by means of a bank of matched filters, considering the interfering users as random noise. However, this approach does not take into account the information content in the interfering signals. A multi-user detector (MUD) exploits this information in order to achieve a higher system capacity. Within the MUD family, interference cancellation schemes have an important role since they achieve a good performance with a lower complexity. In addition, another class of techniques applicable in return link can be classified as static, semi-static, and dynamic inter-cell interference coordination. In the following, these techniques are elaborated, and the main outcomes of the performance assessment are reported.

Multi-user detection: The following MUD techniques are studied: linear techniques, such as zero-forcing and linear MMSE filtering, successive interference cancellation (SIC), and a multi-branch decision feedback (MB) technique. MB exploits different ordering patterns to improve the performance of a SIC algorithm, and a maximum-likelihood detector makes the final decision over the list of candidates generated by the multiple branches. The ordering patterns are obtained from permutation of the first branch ordering which can be based on complex channel gain ordering (MB-CG) or best output signal-to-noise-andinterference ratio ordering (MB-SNIR). MB-CG achieves similar results as SIC with optimum ordering (SIC-SNIR), while requiring a significantly lower complexity, whereas MB-SNIR can achieve a gain of $3-5 \mathrm{~dB}$ in interference-limited scenarios such as in the case of FR-1, but it does not improve significantly the performance of SIC-SNIR in case of FR-4 and FR-2. Nevertheless, at low SNR (less than $10 \mathrm{~dB}$ ), all the considered techniques show similar performance, therefore LMMSE becomes a good low-complexity candidate for noise-limited scenarios as shown in Fig. 5 a), where spectral efficiency improvements achieved with LMMSE and MB-CG are reported.

MUD techniques are also sensitive to channel estimation errors but the diversity achieved through the MB approach allows to counteract the non-ideal channel state information (CSI) better than the other techniques [12]. As reported in Fig. 5 b) for FR-2, MB with training sequence $\mathrm{L}=24$ has the same MSE as V-BLAST with training sequence $\mathrm{L}=48$ for SNR greater than $15 \mathrm{~dB}$.

Interference cancellation: Interference cancellation (IC) techniques have been studied in the considered scenario extending the analysis also to lower frequency reuse factors and a variable number of collaborating gateways. In particular, one of the selected techniques is the MMSE successive interference cancellation (MMSE-SIC), where each iteration is composed of a spatial MMSE filtering followed by the demodulation and decoding of the user terminal having 


\begin{tabular}{|c|c|c|c|c|c|c|c|}
\hline \multicolumn{9}{|c|}{ FR=4,8GW } \\
\hline $\begin{array}{c}\text { Reference } \\
\text { overall } \\
\text { Es/No }\end{array}$ & $\begin{array}{c}\text { Reference SE } \\
\text { [bits/symbol] }\end{array}$ & $\begin{array}{c}\text { Es/N0 } \\
\text { gain }\end{array}$ & New SE & $\begin{array}{c}\text { SE gain } \\
{[\%]}\end{array}$ & $\begin{array}{c}\text { Es/N0 } \\
\text { gain }\end{array}$ & New SE & $\begin{array}{c}\text { SE gain } \\
{[\%]}\end{array}$ \\
\hline 0.2 & 0.609 & 5 & 0.931 & 52.87 & 5 & 0.931 & 52.87 \\
\hline 3.2 & 0.931 & 4.55 & 1.307 & 40.39 & 4.51 & 1.307 & 40.39 \\
\hline 4.8 & 1.307 & 2.64 & 1.475 & 12.85 & 2.68 & 1.475 & 12.85 \\
\hline 5.9 & 1.475 & 1.98 & 1.649 & 11.8 & 2.03 & 1.649 & 11.8 \\
\hline 7 & 1.649 & 1.55 & 1.649 & 0 & 1.64 & 1.649 & 0 \\
\hline 9.3 & 1.757 & 1.22 & 1.98 & 12.69 & 1.4 & 1.98 & 12.69 \\
\hline 10.4 & 1.98 & 1.19 & 1.98 & 0 & 1.42 & 1.98 & 0 \\
\hline 12.1 & 2.198 & 1.32 & 2.668 & 21.38 & 1.59 & 2.668 & 21.38 \\
\hline 13.1 & 2.668 & 1.37 & 2.965 & 11.13 & 1.69 & 2.965 & 11.13 \\
\hline 14.5 & 2.965 & 1.65 & 2.965 & 0 & 2.01 & 2.965 & 0 \\
\hline
\end{tabular}

(a)

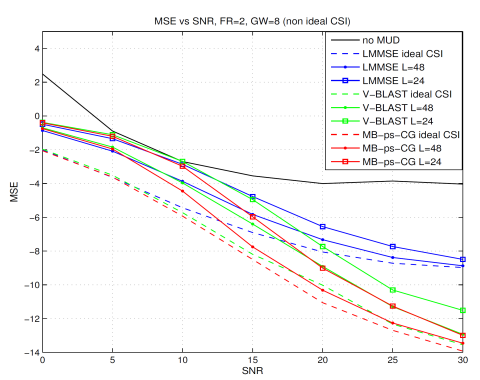

(b)

Fig. 5: a) $E_{\mathrm{s}} / N_{0}$ and SE improvement with MUD, FR-4, 8 GWs. b) MSE vs. SNR, FR-2, 8 GWs.

\begin{tabular}{|c|c|c|c|c|c|c|c|}
\hline \multirow[b]{2}{*}{$\begin{array}{c}\text { Reference } \\
\text { overall } \\
\text { Es/No } \\
\text { [dB] }\end{array}$} & \multirow[b]{2}{*}{\begin{tabular}{|c} 
Reference \\
SE \\
[bits/symbol]
\end{tabular}} & \multicolumn{3}{|c|}{ MMSE-SIC } & \multicolumn{3}{|c|}{ MMSE-CPIC } \\
\hline & & $\begin{array}{c}\text { Es/No } \\
\text { gain } \\
{[\mathrm{dB}]}\end{array}$ & $\begin{array}{c}\text { New SE } \\
\text { [bits/symbol] }\end{array}$ & $\begin{array}{c}\text { SE gain } \\
{[\%]}\end{array}$ & $\begin{array}{c}\text { Es/No } \\
\text { gain } \\
\text { [dB] }\end{array}$ & $\left|\begin{array}{c}\text { New SE } \\
\text { [bits/symbol] }\end{array}\right|$ & $\begin{array}{c}\text { SE gain } \\
{[\%]}\end{array}$ \\
\hline 0,2 & 0,609 & 5,51 & 0,931 & 52,87 & 5,47 & 0,931 & 52,87 \\
\hline 3,2 & 0,931 & 5,51 & 1,307 & 40,39 & 5,47 & 1,307 & 40,39 \\
\hline 4,8 & 1,307 & 2,78 & 1,475 & 12,85 & 2,85 & 1,475 & 12,85 \\
\hline 5,9 & 1,475 & 2,12 & 1,649 & 11,8 & 2,23 & 1,649 & 11,8 \\
\hline 7 & 1,649 & 1,67 & 1,649 & 0 & 1,81 & 1,649 & 0 \\
\hline 9,3 & 1,757 & 1,46 & 1,98 & 12,69 & 1,61 & 1,98 & 12,69 \\
\hline 10,4 & 1,98 & 1,44 & 1,98 & 0 & 1,62 & 2,198 & 11,01 \\
\hline 12,1 & 2,198 & 1,56 & 2,668 & 21,38 & 1,74 & 2,965 & 34,9 \\
\hline 13,1 & 2,668 & 1,65 & 2,965 & 11,13 & 1,8 & 2,965 & 11,13 \\
\hline 14,5 & 2,965 & 1,97 & 2,965 & 0 & 2,12 & 2,965 & 0 \\
\hline
\end{tabular}

(a)

\begin{tabular}{|l|c|}
\hline Scenario (0.7 IUR) & $\begin{array}{c}\text { Capacity } \Delta \% \\
\text { wrt to unscheduled scenario }\end{array}$ \\
\hline \hline Unscheduled & 0 \\
\hline Scheduled - Min & 43.25 \\
\hline Scheduled - Average & 109.5 \\
\hline Scheduled - Max & 147.26 \\
\hline
\end{tabular}

(b)

Fig. 6: a) $E_{\mathrm{s}} / N_{0}$ and SE improvement with IC, FR-4, 8 GWs. b) Return link capacity with and without scheduling, FFR scenario.

the highest value of SNIR [13]. In addition, MMSE filters can be also applied to the convention parallel interference cancellation (CPIC) technique [14].

The gains in terms of spectral efficiency and required $E_{\mathrm{s}} / N_{0}$ in case of 8 cooperating gateways are shown in Fig. 6 a). MMSE-SIC and MMSE-CPIC have confirmed the results achieved in terms of MSE [9] also in terms of required SNR and spectral efficiency improvements.

Interference coordination Interference coordination (ICIC) schemes [15], such as FFR with scheduling to coordinate the users transmissions in the frequency and time domain, can also improve the SNIR performance and spectral efficiency. An average carrier-to-interference ratio (C/I) improvement of $3 \mathrm{~dB}$ is observed for the users in the outer beam, while the improvement is greater than $3 \mathrm{~dB}$ for the users inside the inner beam [16]. The resulting capacities for the FFR scenario with an inner beam user ratio (IUR) equal to 0.7 (with $50 \%$ more bandwidth per beam as compared to FR-4), reported in Fig. 6 b), show an average improvement in the return link capacity of $109.5 \%$. 


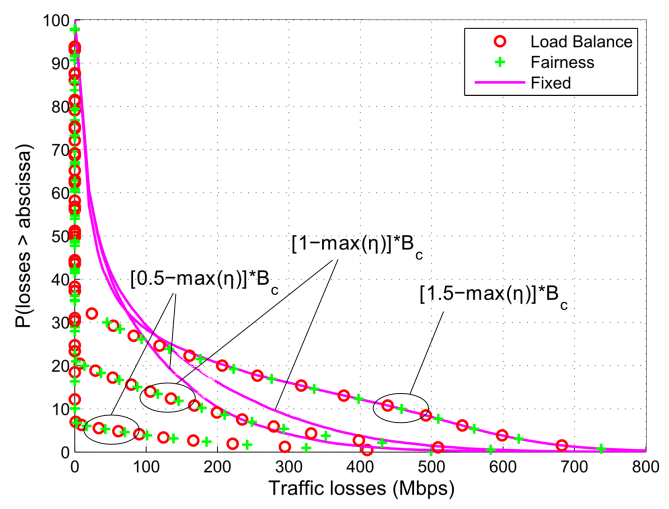

Fig. 7: CCDF of traffic losses for a fixed scheduling and the proposed algorithms for 4 gateways in a cluster.

\section{Radio resource management}

Interference-aware RRM is considered as the key to the realization of very high resource reuse HTS systems. In the forward link, if the system is under full load, i.e. all the beams are active on all the resources, the CCI experienced by an intended user is constant and cannot be alleviated through scheduling. However, if there are free resources, users with low carrier-to-noise ratio $(\mathrm{C} / \mathrm{N})$ can be scheduled on resources which are lightly used across the beams. Alternatively, a number of interfering beams can be switched off at a given time step. In the return link, based on the association of users in the co-channel beams to share a system resource (even at full load), different CCI is experienced by the users. Through optimization of the user associations and scheduling over the available resources, the CCI can be minimized and the user throughput maximized.

\subsection{Satellite-switched smart gateway diversity for feeder link}

Considering a time switched payload, more flexibility in terms of interconnections between feeder and user links is introduced into the system. The time that each feeder link is connected with a user link may vary over time. A framework which takes into account the atmospheric conditions of each gateway plus the traffic demands of the beams and decides the number of time slots that each gateway is connected to a user beam is proposed allowing a match of the instantaneous user demands with the offered capacity of the gateways. The dwelling time of a gateway to a user beam can be tuned such as to minimize the capacity losses, keeping as low as possible the number of gateways in the system whilst providing the required availability. Two objective functions have been considered herein: 1) one that maximises the product of the satisfaction ratios (offered traffic/traffic demands) for the sake of load balance; and 2) another that maximises the minimum satisfaction ratio for the sake of fairness. Fig. 7 shows the complementary CDF of the traffic losses for three traffic scenarios, where the users 


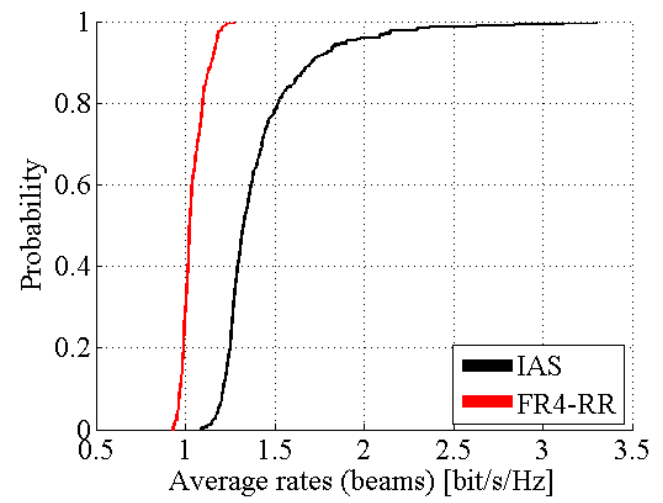

Fig. 8: Average rate per beam in the BATS scenario.

traffic demands are drawn from a uniform distribution $[\{0.5,1,1.5\}-\max (\eta)]^{*} B_{c}$, considering 4 gateways in a cooperation cluster. The maximum achievable spectral efficiency, $\max (\eta)$, depends on the link budget, detailed for the BATS system in [17] for $B_{\mathrm{c}}=447.3 \mathrm{MHz}$. An important observation is the reduction of traffic losses due to the better match of feeder transmissions rates with the user beam demands. The effectiveness of the proposed methods is improved as the number of gateways in the cluster or the traffic variance increase.

\subsection{Interference-aware scheduling for forward user link}

The IAS algorithm [18] for full reuse in the forward link utilizes partial channel state information (CSI) to reduce the number of interfering beams on the served users. Neighbouring beams are switched off, if a served user cannot handle the interference caused by these beams. As a result, it is best suitable for burst mode beam hopping applications. The performance of the IAS is compared to a FR4 Round Robin scheme. A noise power of $-82.4 \mathrm{dBm}$ and a transmit power of $47.4 \mathrm{dBm}$ per beam are assumed. The clear-sky received power of the users' strongest beam varies between $-74 \mathrm{dBm}$ and $-64.7 \mathrm{dBm}$ due to path loss and antenna gains of transmit and receive antenna. The user rates $R$ are calculated by the Shannon function. The system rate is calculated by averaging the rates of the beams normalized to the full system bandwidth of $2.9 \mathrm{GHz}$ in the $\mathrm{Ka}$ band forward user link. In Fig. 8, the CDF of the average rate of the beams is presented. The IAS is able to increase the minimum, maximum and mean data rate of the all users. Since TDM is employed, disabling of beams does not relate to an outage, but rather a delay in serving the traffic request. However, no noteworthy latency is observed compared to the FR4-RR. Both the IAS and the FR4-RR presented no SNIR values below the outage threshold of $-1.85 \mathrm{~dB}$ defined in the BATS system, therefore meeting the availability requirement of $99.7 \%$. The IAS is able to achieve an average system rate of $1.415 \mathrm{bit} / \mathrm{s} / \mathrm{Hz}$, while the FR4-RR only achieves an average system rate of $1.043 \mathrm{bit} / \mathrm{s} / \mathrm{Hz}$, which represents a gain of $35.7 \%$. 


\begin{tabular}{|c|c|c|c|c|c|}
\hline \multirow{2}{*}{ FR } & \multirow{2}{*}{$\mathrm{SIC}$} & \multirow{2}{*}{ Scheduling } & \multicolumn{2}{|c|}{ Capacity [Gbps] } & \multirow{2}{*}{ Availability } \\
\hline & & & Value & Increase & \\
\hline 4 & $\mathrm{No}$ & Random & 384.6 & - & $99.99 \%$ \\
\hline \multirow{10}{*}{1} & \multirow{5}{*}{ No } & Random & 575.7 & $49.7 \%$ & $98.92 \%$ \\
\hline & & Distinct & 585.2 & $52.2 \%$ & $99.97 \%$ \\
\hline & & Distinct FSA & 578.4 & $50.4 \%$ & $99.97 \%$ \\
\hline & & Common & 596 & $55 \%$ & $99.97 \%$ \\
\hline & & Common FSA & 586.7 & $52.5 \%$ & $99.97 \%$ \\
\hline & \multirow{5}{*}{ Yes } & Random & 746.2 & $94 \%$ & $99.99 \%$ \\
\hline & & Distinct & 762 & $98.1 \%$ & $99.99 \%$ \\
\hline & & Distinct FSA & 742.9 & $93.2 \%$ & $99.99 \%$ \\
\hline & & Common & 766 & $99.2 \%$ & $99.99 \%$ \\
\hline & & Common FSA & 746.8 & $94.2 \%$ & $99.99 \%$ \\
\hline
\end{tabular}

Table 2: System capacity and availability improvements.

\subsection{Scheduling based on multi-partite graph matching for return user link}

Scheduling algorithms for full reuse based on multi-partite graph matching that maximize the minimum user rates have been presented in [19]. To reduce the computational complexity, users are split into clusters. The assumption is made that users in second tier beams contribute only negligibly to the CCI. Therefore, a virtual 4-color scheme is applied, and the users in a color are scheduled randomly. These random cluster schedules are merged together by applying a minimum deletion algorithm and graph matching techniques to find the optimum user association. The low-complexity distinct merging is a greedy approach that concatenates the clusters sequentially. The globally optimum common approach merges all clusters in one step. A variation of both techniques is the free slot assignment (FSA) method, where one slot per schedule is left unallocated to increase the flexibility in the scheduler. In this study, the scheduling performance is evaluated with and without the application of SIC at the gateway. A bandwidth of $1.05 \mathrm{GHz}$ in Ka band is considered in 302 beams over Europe, and the DVB-RCS2 MODCODs are applied. The results for the capacity and availability improvements are summarized in TABLE 2. It has to be noted that either scheduling or SIC need to be employed with full reuse so that the system meets the availability requirement of $99.7 \%$. In terms of system capacity, the common merging gives the best results, i.e. the average system capacity is increased by $2.7 \%$ in the case with SIC and by $3.5 \%$ in the case without SIC as compared to the random scheduling method. The distinct method offers a good compromise between performance and computational complexity. In the case with SIC, the FSA algorithm has a performance comparable to the random scheduling, while it is slightly better in the case without SIC. However, it should be noted that the full frequency reuse system with scheduling and SIC is able to double the capacity as compared to the state-of-the-art 4-color scheme with random scheduling, while it still offers more than $50 \%$ increase, when SIC is not employed. 


\section{Conclusion}

In this paper, several capacity enhancing techniques have been evaluated for application in HTS communication systems. In the physical layer, non-linear predistortion and equalization techniques are essential to preserve the spectral efficiency gains of higher order modulations when high baudrates and very low roll-off factors are employed. Performance gains in terms of SIR improvement in the range from $7.3 \mathrm{~dB}$ to $12 \mathrm{~dB}$ have been reported. The application of FFTbased waveforms in the return link has shown the highest spectral efficiency in the non-linear channel, especially when an IMI cancelling receiver is employed, whereby SC-FDMA demonstrated an overall power efficiency gain of more than 2 $\mathrm{dB}$ as compared to state-of-the-art TDMA. GPS-based delay pre-compensation has been shown to enable synchronization acquisition with negligible $\mathrm{CP}$ overhead. Timing and frequency offsets are best estimated and tracked by means of the M\&M algorithm and the Moose algorithm, respectively, showing significant MSE improvement even at low SNR. The adoption of interference management techniques in multi-beam satellite systems allows the use of more aggressive frequency reuse patterns and provides promising improvements in terms of available system bandwidth and total throughput. CCI-aware RRM is shown to be an enabling factor for realization of full resource reuse HTS communication systems. Satellite-switched smart gateway diversity with scheduling helps to reduce the total number of gateways in the system, while ensuring the necessary feeder link availability in rain fading. The IAS approach for full frequency reuse in the forward link and the scheduling based on multi-partite graph matching in the return link respectively offer $35.7 \%$ and $99.2 \%$ increase of the system capacity as compared to state-of-the-art 4-color reuse with random scheduling.

\section{Acknowledgement}

This work has been supported by the BATS research project funded by the EU-FP7 under contract n317533.

\section{References}

1. Broadband Access via Integrated Terrestrial and Satellite Systems (BATS), ICT2011.1.1 BATS D4.1: Satellite Network Mission Requirements, European Project, Tech. Rep., (2012)

2. Ding, L., Zhou, G. T., Morgan, D. R., Zhengxiang, M., Kenney, J. S., Jaehyeong, K., Giardina, C. R.: A Robust Digital Baseband Predistorter Constructed Using Memory Polynomials, In: IEEE Transactions on Communications, vol. 52, no. 1, pp. 159-165, Jan. (2004)

3. Technical Specifcation Group Radio Access Network; Evolved Universal Terrestrial Radio Access (EUTRA); Physical Layer Procedures (Rel. 11), 3rd Generation Partnership Project Std. 3GPP TS 36.213, (2013) 
4. Mengali, U., Morelli, M.: Data-aided Frequency Estimation for Burst Digital Transmission, In: IEEE Transactions on Communications, vol. 45, no. 1, pp. 23-25, Jan. (1997)

5. Moose, P. H.: A Technique for Orthogonal Frequency Division Multiplexing Frequency Offset Correction, In: IEEE Transactions on Communications, vol. 42, no. 10, pp. 2908-2914, Oct. (1994)

6. Benfatto, D., Privitera, N., Suffritti, R., Awoseyila, A., Evans, B., Dimitrov, S.: On Acquisition and Tracking Methods for SC-FDMA over Satellite, IN: Proc. of 7th Advanced Satellite Multimedia Systems Conference (ASMS2014), Livorno, Italy, Sep. 8-10 (2014)

7. Dimitrov, S., Boccolini, G., Jaeckel, S., Benfatto, D., Privitera, N., Suffritti, R., Awoseyila, A., Evans, B.: FFT-based Waveforms for High Through Satellite Communications: Opportunities and Challenges, IN: Proc. of 20th Ka and Broadband Communications Conference, Vietri sul Mare / Salerno, Italy, Oct. 1-3 (2014)

8. Dimitrov, S., Privitera, N., Suffritti, R., Boccolini, G., Awoseyila, A., Evans, B.: Spectrally Efficient Waveforms for the Return Link in Satellite Communication Systems, In: Proc. of European Conference on Networks and Communications 2015 (EUCNC2015), Paris, France, Jun. 29-Jul. 2 (2015)

9. Suffritti, R., Privitera, N., Dimitrov, S., Katona, Z., Boccolini, G., Jaeckel, S., Raschkowski, L., Kyrgiazos, A., Evans, B., Rodriguez, J. M., Yun, A., Vidal, O., Inigo, P.: On Interference Management Techniques in Broadband Satellite Systems, In: Proc. of Ka Conference, (2014)

10. Liu, J., Krzymien, W.: A novel nonlinear precoding algorithm for the downlink of multiple antenna multi-user systems, In: Proc. of IEEE Vehicular Technology Conference, vol. 2, pp. 887-891, (2005)

11. Chang, R. Y., Tao, Z., Zhang, J., Kuo, C.-C. J.: Dynamic fractional frequency reuse (D-FFR) for multicell OFDMA networks using a graph framework, In: Wireless Commun. Mob. Comput., (2011)

12. Arnau, J., Mosquera, C.: Multiuser detection performance in multibeam satellites links under imperfect CSI, In: Proc. of Asilomar, (2012)

13. Gallinaro, G., Debbah, M., Muller, R., Rinaldo, R., Vernucci, A.: Interference mitigation for the reverse-link of interactive satellite networks, In: Proc. of 9th International Workshop on Signal Processing for Space Communications, (2006)

14. Chan, A., Wornell, G. W.: A class of asymptotically optimum iterated-decision multiuser detectors, In: Proc. of IEEE International Conference on Acoustics, Speech, and Signal Processing (ICASSP '01), vol. 4, pp. 2265-2268, (2001)

15. Pateromichelakis, E., Shariat, M., Quddus, A., Tafazolli, R.: On the Evolution of Multi-Cell Scheduling in 3GPP LTE / LTE-A, In: IEEE Communications Survey and Tutorials, (2012)

16. Ng, U. Y., Kyrgiazos, A., Evans, B.: Interference Coordination for the Return Link of a Multibeam Satellite System, In: Proc. of 7th Advanced Satellite Multimedia Systems Conference, (2014)

17. Dimitrov, S., Erl, S., Jaeckel, S., Rodriguez, J. M., Yun, A., Kyrgiazos, A., Evans, B., Vidal, O., Inigo, P.: Radio Resource Management for Forward and Return Links in High Throughput Satellite Systems, In: Proc. of 20th Ka and Broadband Communications Conference, Vietri sul Mare / Salerno, Italy, Oct. 1-3, (2014)

18. Dimitrov, S., Erl, S., Barth, B., Jaeckel, S., Kyrgiazos, A., Evans, B.: Radio Resource Management Techniques for High Throughput Satellite Communication Systems, In: Proc. of European Conference on Networks and Communications 2015 (EUCNC2015), Paris, France, Jun. 29-Jul. 2 (2015) 
19. Boussemart, V., Berioli, M., Rossetto, F.: User scheduling for large multi-beam satellite MIMO systems," in In Proc. of the IEEE Asilomar Conference on Signals, Systems and Computers 2011 (ASILOMAR 2011), pp. 1800-1804, (2011) 